develop particularly severe bone fragility accompanied with high risk factors for fragility fractures ${ }^{1,2}$. Although bisphosphonate is recommended for treatment of osteoporosis related RA and glucocorticoid-induced-osteoporosis ${ }^{3}$, there are more frequently fractures than expected from bone mineral density based prediction even after used bisphosphonate.

Objectives: To clarify the pathological mechanisms of bone fragility of these RA patients, we investigated bone biopsy samples obtained from RA and normal postmenopausal patients.

Methods: We examined 10 female postmenopausal RA patients receiving glucocorticoid and bisphosphonate therapy (RA group) and 10 age-matched female patients with postmenopausal osteoporosis (Ctl group) selected from patients who required autologous iliac bone grafts. Analyses of clinical data, bone mineral density, serum metabolic markers, bone quality and material mechanical property of biopsy sample were performed.

Results: Although bone mineral density didn't show significant differences, RA group had significantly higher score of fracture risk assessment tool (FRAX), number and severity of existing vertebral fractures.

\begin{tabular}{lccc}
\hline & Control & RA & $p$ \\
\hline FRAX score (\%) & & & \\
$\quad$ Major osteoporosis fx. & $10.7 \pm 4.8$ & $33.4 \pm 8.0$ & $<0.001$ \\
$\quad$ Femoral neck fx. & $2.8 \pm 2.4$ & $9.9 \pm 6.1$ & 0.002 \\
Clinical fragility fx. & 4 & 16 & \\
$\quad$ Number & 4 & 29 & \\
$\quad$ Severity (\# $x$ grade) & $218.8 \pm 24.6$ & $164.9 \pm 36.5$ & 0.001 \\
$\quad$ Mechanical strength (N/mm) &
\end{tabular}

RA group exhibited significant bone quality abnormalities including deterioration of the bone microstructure, decreased calcification of the bone matrix, increased osteocyte atrophy and empty lacunae (Figure), and an impairment bone material strength properties.
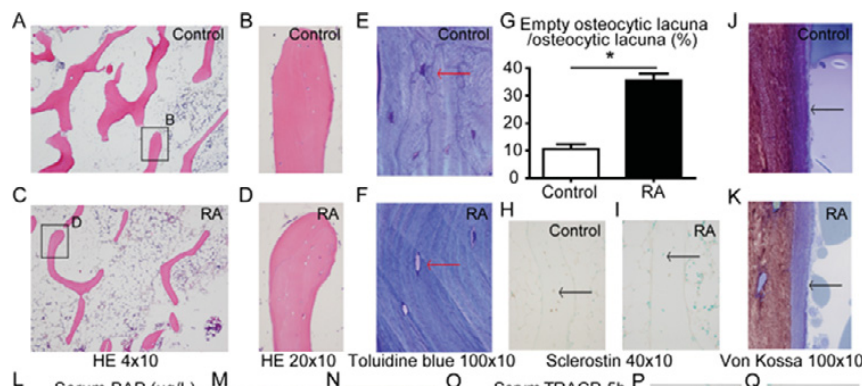

L Serum BAP $(\mu g / L)$
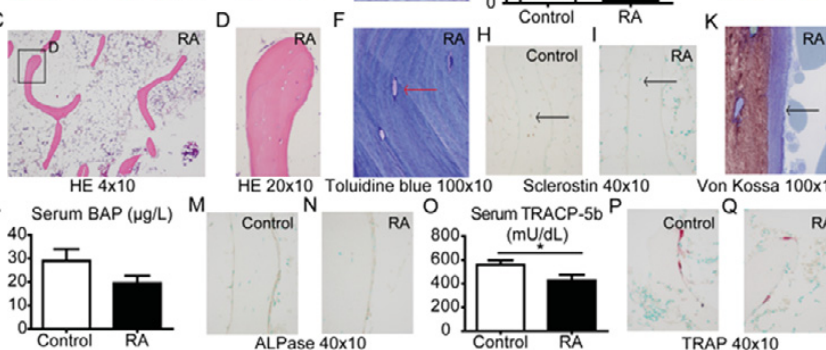

Figure: Histological mechanism of deterioration of bone quality in RA patients receiving GCs. (A-D) Representative photomicrographs of HE stained sections of the specimen. Images show sections from Control (A, C) and RA groups (B, D). Boxed areas in B and D are shown at higher magnification in the indicated images. (E, F) Representative micrographs focused on osteocytes of Control (E) and emptiness in RA patients. (G) Comparison of ratio of empty osteocytic lacuna is shown. (H, I) Representative micrographs of Control (H) and RA groups (I) with immunostaining of sclerostin (black arrow). (J, K) Representative micrographs of Control (J) and RA group (K) with von Kossa staining. Black arrows show the cement lines. (L) Comparison of serum BAP is shown. (M, N) Representative micrographs of Control (M) and RA groups (N) with immunostaining of sclerostin ALP ase. (O) Comparison of serum TRACP-5b is shown. (P, Q) Representative micrographs of Control $(\mathrm{P})$ and RA group $(\mathrm{Q})$ with TRAP staining. Osteoclasts are stained red. Values shown are means \pm SD $(* p<0.05)$.

Conclusions: Our findings showed that RA patients receiving glucocorticoid treatment have severe bone fragility regardless of increased bone quantity by using bisphosphonate. The functional deteriorations of the osteocyte system and the abnormalities of bone quality might induce bone fragility fracture. Therefore, management of osteoporosis associated with RA should be targeted about bone quality as well as bone quantity.

References:

[1] Kanis JA et al. J Bone Miner Res 2004

[2] Takaha M et al. Arthritis Rheum 2012.

[3] Grossman, J.M. et al. Arthritis Care Res 2010.

Acknowledgements: This project was supported in part by a Grant-in-Aid for Scientific Research (C) from the Ministry of Education, Culture, Sports, Science, and Technology of Japan 25462357 (M. Takahata).

Disclosure of Interest: None declared

DOI: 10.1136/annrheumdis-2017-eular.3514

\section{FRI0076 LACTATE/SLC5A12-INDUCED METABOLIC SIGNALLING NETWORK: A NEW TARGET IN RHEUMATOID ARTHRITIS}

V. Pucino ${ }^{1,2}$, J. Smith $^{1}$, D. Cucchi ${ }^{1}, \mathrm{~K}$ Blighe $^{3}$, M. Bombardieri ${ }^{2}$, C. Pitzalis ${ }^{2}$ C. Mauro ${ }^{1}$. ${ }^{1}$ Centre of Biochemical Pharmacology; ${ }^{2}$ Centre for Experimental Medicine and Rheumatology, William Harvey Research Institute, Barts and The London SMD, Queen Mary University of London, London, United Kingdom; ${ }^{3}$ Channing Division of Network Medicine, Brigham and Women's Hospital and Harvard Medical School, Boston, United States

Background: RA is a systemic-autoimmune-disease characterized by chronic inflammation of the synovial-joints. Up to $30-40 \%$ of patients do not respond to treatment and current biomarkers are largely insensitive at predicting disease progression and response to treatment. It is well recognised that RA-synovitis is a heterogeneous pathology with different histological phenotypes i.e. lymphoid- $(L)$, myeloid- $(\mathrm{M})$ and fibroid- $(\mathrm{F})$. As a result of inflammation the RA synovial microenvironment is hypoxic and acidic partly due to the accumulation of lactate, the end product of anaerobic glycolysis. Exposure of $\mathrm{CD}^{+} \mathrm{T}$ cells to lactate inhibits their migratory capabilities and induces their shift toward a Th17 phenotype $e^{1,2}$. These effects are mediated via the interaction of lactate with its transporter SLC5A12, which is expressed on the CD4 ${ }^{+}$T cell surface ${ }^{1,2}$.

Objectives: To characterize whether the newly identified lactate/SLC5A12induced metabolic signalling pathway can be harnessed in the stratification of RA patients in the different histological phenotypes and to modulate inflammatory responses in vitro and in vivo.

Methods: RA peripheral blood cells, RA synovial tissues (ST, 21 lymphoid and 8 myeloid) and mononuclear cells from tonsil of patients undergoing tonsillectomy were included in the analysis. RNA sequencing of RA-ST was performed and data analysed for the expression of metabolic genes. SLC5A12 expression and IL17 production was performed by flow-cytometry. Cytokines and transcription factors mRNA relative expression was evaluated by RT-PCR. Seahorse and western blot analysis was performed for the evaluation of metabolic pathways. Transwell plates were used for migration assays. Human-glucose-6-phosphate-isomerase (hG6PI) induced arthritis model was used to evaluate the impact of anti-SLC5A12 on the clinical and histological score.

Results: We showed that: i) the expression of SLC5A12 is up-regulated by CD4+ T cells upon inflammation; ii) SLC5A12 is up-regulated in anti-CD3 stimulated RA CD4 ${ }^{+} \mathrm{T}$ cells cultured in autologous synovial fluid; iii) the lactate/SLC5A12 induced metabolic pathway is differentially activated in RA patients with distinctive synovial "pathotypes"; iv) SLC5A12 antibody reduces lactate-induced pro-inflammatory cytokines, limits Th17 and follicular helper T cell differentiation, reverses lactate impaired $\mathrm{CD}^{+}{ }^{+} \mathrm{T}$ cell migration and restores lactate-mediated inhibition of glycolysis in vitro; v) antibody-mediated blockade of SLC5A12 ameliorates the clinical course in human-glucose-6-phosphate-isomerase (hG6PI)-induced arthritis.

Conclusions: Targeting lactate/SLC5A12-induced metabolic signalling pathway may provide a novel therapeutic strategy to reduce inflammation in RA patients. References:

[1] Haas R. et al. PLoS Biology 2015.

[2] Pucino V. et al. Eur J Immunol 2017

Acknowledgements: This work is supported by a fellowship from the Arthritis Research UK (ARUK) to V.P. by a fellowship from the British Heart Foundation, a project grant from the CARIPLO Foundation, a Proof of Concept award from Queen Mary Inno-vation, Ltd., to C.M. and MRC ARUK founded project "The Pathobiology of Early Arthritis Cohort (PEAC)" to C.P.

Disclosure of Interest: None declared

DOI: 10.1136/annrheumdis-2017-eular.3993

\section{FRI0077 GUT COMMENSALS MODULATE INFLAMMATION VIA T REGULATORY CELLS}

\section{Taneja. Mayo Clinic, Rochester, United States}

Background: Role of environmental factors in predisposition to develop rheumatoid arthritis (RA) have gained interest due, in part, to the studies showing an association of gut microbiota with immune homeostasis. Although the etiology of RA is unknown, recent studies on the role of gut microbiota in inflammatory adaptive immune response have led to the concept that interaction between the host microbiome and genetic factors influences autoimmunity. We have recently shown an association of Collinsella aerofaciens with RA

Objectives: In this study, we aimed to determine how human gut commensals modulate arthritis phenotype in humanized mice expressing RA-susceptible HLA-DQ8.

Methods: DQ8 mice following immunization with type II collagen develop arthritis and antigen-specific cellular and humoral response. DQ8 mice orally gavaged with RA-associated Collinsella aerofaciens and non-associated Prevotella histicola on alternate days for one week and were induced for arthritis. Gavage with microbes continued for 4 weeks. Mice were monitored for onset and progression of arthritis. Th17 regulatory network and cells involved in regulation were analyzed. DQ8 mice were gavaged with fecal homogenates supernatants from arthritis-resistant mice, induced for arthritis and monitored for disease.

Results: Mice gavaged with $C$. aerofaciens enhanced disease severity while $P$. histicola protected mice from arthritis. While treatment with $P$. histicola reduced intestinal permeability by increasing expression of tight junction proteins, C. 